\title{
ASYMPTOTIC VALUES OF HYPERBOLIC MONOPOLES
}

\author{
PAUL NORBURY
}

\begin{abstract}
We show that many hyperbolic monopoles can be distinguished from each other via their asymptotic values in contrast to the case of Euclidean monopoles.
\end{abstract}

\section{INTRODUCTION}

Magnetic monopoles initially arose out of Dirac's study of the quantum theory of electro-magnetism. They are singular solutions of Maxwell's equations valid away from their singularities. Bogomolny-Prasad-Sommerfield monopoles are a generalisation of Dirac monopoles to non-abelian theories where the singularities can be smoothed away. They are solutions to the equation $d_{A} \Phi=* F_{A}$ where $(A, \Phi)$ is a pair given by a connection with $L^{2}$ curvature $F_{A}$ and a Higgs field-a section of the adjoint bundle - defined on a trivial bundle over $\mathbb{R}^{3}$ with structure group a compact Lie group $G$, and the Hodge star is given with respect to a metric on $\mathbb{R}^{3}$. The Higgs field $\Phi$ is constrained to lie in a given orbit of the Lie algebra on the sphere at infinity. The mass of the monopole is defined to be the conjugacy class of the Higgs field on the sphere at infinity, or more generally it is the orbit in the Lie algebra under the action of the group of the Higgs field on the sphere at infinity. The mass also defines an embedding of the circle (the gauge group of the abelian theory) into the group $G$ so that BPS monopoles can be compared to Dirac monopoles.

It is reasonable to ask what BPS monopoles look like from a long distance, and to the extreme, on the sphere at infinity. It is not necessarily true that they should look like Dirac monopoles since the latter approximate BPS monopoles not only far from the singularities but also only when the singularities are far apart. We can ask this question for different metrics on $\mathbb{R}^{3}$. When the metric is Euclidean, the monopoles on the sphere at infinity do look exactly like Dirac monopoles. Moreover, as for Dirac monopoles, up to charge, all Euclidean monopoles look the same at infinity.

In this paper we will consider hyperbolic monopoles defined over hyperbolic space $\mathbb{H}^{3}$. We will show that on the sphere at infinity, the BPS hyperbolic monopoles take on many different values in contrast to the Euclidean case. This agrees with the conjecture that in fact hyperbolic monopoles are determined by their values on the sphere at infinity. This conjecture has been confirmed for integral mass $S U(2)$ hyperbolic monopoles by Austin and Braam [2] and it is an easy fact for hyperbolic Dirac monopoles. The proof of the integral mass $S U(2)$ case by Austin and Braam in uses some beautiful algebraic geometry and introduces discrete Nahm data. This approach has been generalised to $S U(n)$ by Murray and Singer 15 and it is likely this will lead to a proof that these monopoles are determined by their asymptotic

1991 Mathematics Subject Classification. 81T13, 53C07. 
values. Our aim is to complement this work with proofs that work for all gauge groups and non-integral mass. We are also interested in how such proofs fail for Euclidean monopoles.

Murray and Singer [16] also study the twistor theory of hyperbolic monopoles. Their results hold for any mass since rather than working with circle invariant instantons over the four-sphere which requires integral mass they work with instantons over Minkowski space invariant under translations. They show that a hyperbolic monopole is determined by its asymptotic value plus some extra information (remark (3) on p.989.)

It may end up that methods of algebraic geometry will be needed to show that a hyperbolic monopole is determined by its asymptotic value. We believe the main result of this paper is still valuable since it directly shows why hyperbolic monopoles and Euclidean monopoles behave differently.

As mentioned, a monopole has a mass given by an element of the Lie algebra, or really the orbit of the element. We can parametrise the moduli space of monopoles with given mass by holomorphic maps from the two-sphere into the orbit in the Lie algebra - see Section 3. Since many different masses have isomorphic orbits and hence the same parametrisation we can speak of a subset of the moduli space before specifying the mass precisely. This same idea is used in the study of "monopole clouds".

Theorem 1. Given two disjoint compact subsets in the parameter space of monopoles, if the mass is small enough then the asymptotic values of the corresponding hyperbolic monopoles respectively give two distinct subsets.

Remarks: (i) It is probably true that only one of the subsets of the parameter space need be compact. We discuss this in Section 1

(ii) One satisfying aspect of the theorem is that we can see where the proof fails for Euclidean monopoles.

In Section 2 we describe Dirac monopoles over Euclidean and hyperbolic spaces. In Section 3 we give the background to the holomorphic map associated to a monopole. In Section 1 we give the proof of Theorem 11. We contrast properties of the asymptotic values of hyperbolic monopoles with those of Euclidean monopoles in Section 5 .

\section{DiraC MONOPOLES.}

In this section we study the elementary issue of Dirac monopoles since they give an analogue to the non-linear problem. It is interesting to the note that at infinity Euclidean BPS monopoles look exactly like Euclidean Dirac monopoles whereas hyperbolic space detects a difference between BPS monopoles and Dirac monopoles.

A Dirac monopole is a solution to Maxwell's equations: $F_{A}=* d_{A} \Phi$ where $F_{A}$ is the magnetic field, or the curvature of a connection $A$, and $d_{A} \Phi$ is the electric field given by the covariant derivative of the Higgs field. The Hodge star $*$ depends on the metric. It follows that the magnetic field is harmonic, so for the Euclidean metric the magnetic field is given by

$$
B=\frac{\hat{r}}{4 \pi r^{2}}
$$


where $\hat{r}$ represents the imaginary-valued 2 -form

$$
\hat{r}=\frac{2 r^{2} d \bar{w} d w}{\left(1+|w|^{2}\right)^{2}}
$$

and more generally

$$
B=\frac{\widehat{(r-a)}}{4 \pi(r-a)^{2}}
$$

is a monopole at the point $a \in \mathbb{R}^{3}$. As $r \rightarrow \infty, r^{2} B \rightarrow \hat{n}$, the unit normal, which is in particular independent of $a$. Thus, on the sphere at infinity a monopole looks like a symmetric distribution, or the imaginary-valued 2-form, $2 d \bar{w} d w /\left(1+|w|^{2}\right)^{2}$. A collection of $k$ monopoles is simply the sum of $k$ of these and thus gives

$$
F_{A}=\frac{2 k d \bar{w} d w}{\left(1+|w|^{2}\right)^{2}}
$$

on the sphere at infinity.

Thus we see that (except for the charge) Euclidean Dirac monopoles cannot be distinguished from a distance. In contrast with this, the situation is exactly the opposite for hyperbolic Dirac monopoles.

Proposition 2.1. Hyperbolic Dirac monopoles are determined by their asymptotic values.

Proof. A hyperbolic Dirac monopole is a solution of the equation $F_{A}=* d_{A} \Phi$ for the hyperbolic Hodge star. The fundamental solution is

$$
B=\frac{\hat{r}}{4 \pi \sinh ^{2}(r)}
$$

so a more general single Dirac monopole is given by

$$
B_{a}(x)=\frac{\hat{\nu}}{4 \pi \sinh ^{2}(d(x, a))}
$$

where $d(x, a)$ is the hyperbolic distance between a point $x \in \mathbb{H}^{3}$ and a given point $a$ and $\hat{\nu}$ is the unit vector pointing (away from $a$ ) along the geodesic joining $x$ and $a$. The asymptotic value of each of these monopoles is given by the unit outward normal vector of $S_{\infty}^{2}$ scaled by $\lim _{r \rightarrow \infty} \sinh ^{2}(r) / \sinh ^{2}(d(x, a))$ (for $r=d(x, 0)$.) It uniquely determines the monopole since it simply gives the symmetric measure $d \bar{w} d w /\left(1+|w|^{2}\right)^{2}$ transformed by the conformal transformation of $S_{\infty}^{2}$ induced by the isometry of $\mathbb{H}^{3}$ that takes 0 to $a$.

The most general Dirac monopole is a linear combination of these single monopoles. The content of this proposition is to show that the linear combination of conformal transformations of the symmetric measure on $S_{\infty}^{2}$ determines the conformal transformations.

A conformal transformation takes

$$
w \mapsto \frac{a w+b}{c w+d} .
$$

Since the subgroup $S U(2)$ fixes the symmetric measure we need only consider conformal transformations of the form

$$
w \mapsto a_{j}\left(w-w_{j}\right), a_{j} \in \mathbb{R}^{+}, w_{j} \in \mathbb{C} .
$$


The symmetric measure maps to $a_{j}^{2} d \bar{w} d w /\left(1+a_{j}^{2}\left|w-w_{j}\right|^{2}\right)^{2}$ so a general Dirac monopole has measure at infinity given by

$$
\sum_{j} \frac{a_{j}^{2} d \bar{w} d w}{\left(1+a_{j}^{2}\left|w-w_{j}\right|^{2}\right)^{2}}
$$

where there might be repeated appearances of a pair $\left(a_{j}, w_{j}\right)$.

The denominator for (2) is given by $\Pi_{j}\left(1+a_{j}^{2}\left|w-w_{j}\right|^{2}\right)^{2}$. Put $w=x+i y$ and set $y=0$. Then the denominator factorises with factors $\left.x-x_{j} \pm i \sqrt{(} y_{j}^{2}+1 / a_{j}^{2}\right)$. Thus, the measure determines each $x_{j}$ and $y_{j}^{2}+1 / a_{j}^{2}$. (We have analytically continued $x$ to take on complex values.) Similarly, if we set $x=0$ then we get each $y_{j}$ and $x_{j}^{2}+1 / a_{j}^{2}$ so we get each $x_{j}, y_{j}, a_{j}$ and the boundary measure has determined the Dirac monopole.

The space of compactly supported continuous functions on hyperbolic space acts on the symmetric measure $\omega$ on $S^{2}$ by $f \cdot \omega=\int_{\mathbb{H}^{3}} f(x) x \cdot \omega d x$ where we think of $x \in S L(2, \mathbb{C}) / S U(2)$. Atiyah has suggested that this action might be faithful.

Conjecture 1. Let $f \in C_{c}^{0}\left(\mathbb{H}^{3}\right)$, then $f \cdot \omega=0 \Leftrightarrow f \equiv 0$.

The proposition would then fit into a rather natural setting, following from limiting behaviour of such a result.

\section{HOLOMORPHIC MAPS.}

In this section we will describe the holomorphic map of the two-sphere into a homogeneous space associated to a monopole via scattering. First we will give a brief description of the homogeneous spaces.

Let $\xi \in \mathbf{g}$, the Lie algebra of $G$, and let $K_{\xi}=\{g \in G \mid g \cdot \xi=\xi\}$ be the isotropy subgroup of $\xi$ from the adjoint action of $G$. Then the homogeneous space $G / K_{\xi}$ is a complex manifold and we can speak of holomorphic maps into this manifold. This is best seen using the isomorphism $G / K_{\xi} \cong G \cdot \xi \subset \mathrm{g}$.

The tangent space of an adjoint orbit $X=G \cdot \xi \subset \mathbf{g}$ has a nice description. At $\eta=g \cdot \xi \in G \cdot \xi$, the tangent space $T_{\eta} X=[\mathbf{g}, \eta] \subset \mathbf{g}$. It is more convenient to use $\mathbf{g} / \operatorname{ker}[\eta, \cdot] \cong T_{\eta} X$. The isomorphism is given by $u \mapsto[u, \eta]$ for $u \in \mathbf{g}$.

Homogeneous spaces have complex realisations

$$
G / K_{\xi} \cong G^{c} / P_{\xi}
$$

where $P_{\xi}$ is the parabolic subgroup of $G^{c}$ with the further property that $P_{\xi} \cap G=$ $K_{\xi}$. The isomorphism (3) simply says that given any $g \in G^{c}$, there exists $p \in P_{\xi}$ such that $g p \in G$ and $p$ is unique up to $p \mapsto p k$ for $k \in K_{\xi}$. When $G=U(n)$, this is the Gram-Schmidt process.

The complex structure at $\xi$ is given by $J u \equiv i u\left(\bmod \mathbf{p}_{\xi}\right)$ with respect to the trivialisation $T_{\xi} X \cong \mathbf{g} / \operatorname{ker}[\xi, \cdot]$. It is well-defined since given $i u$, there is an element $v$ of $\mathbf{p}_{\xi}$, the Lie algebra of $P_{\xi}$, unique up to an element of $\operatorname{ker}[\xi, \cdot]$ such that $i u+v \in \mathbf{g}$. The complex structure at each point of the orbit $\eta=g \cdot \xi$ is defined similarly.

A map $f: S^{2} \rightarrow G / K_{\xi}$ is holomorphic when its lift $u$ to $G$ (defined locally) satisfies $u^{-1} \partial_{x} u+J u^{-1} \partial_{y} u=0$, or equivalently for $w=x+i y$

$$
u^{-1} \partial_{\bar{w}} u(w) \subset \mathbf{p}_{\xi} .
$$

The adjoint orbit has a natural symplectic structure, compatible with the complex structure to give a Kahler structure, given at $T_{\eta} X$ by $\omega(u, v)=\langle\eta,[u, v]\rangle$ where $\langle\cdot, \cdot\rangle$ is the Killing form. At $T_{\eta} X$, the metric is $g(u, v)=\omega(J u, v)$ for the complex 
structure $J$. There are many other symplectic structures and complex structures that arise less naturally.

The map $f: S^{2} \rightarrow G / K_{\xi}$ pulls back the symplectic form $\omega$ to a two-form over $S^{2}$ via its lift $u$ :

$$
f^{*} \omega=\left\langle\xi,\left[u^{-1} \partial_{w} u, u^{-1} \partial_{\bar{w}} u\right]\right\rangle d \bar{w} d w .
$$

A hyperbolic monopole $(A, \Phi)$ with finite energy, $\left\|F_{A}\right\|_{2}<\infty$, has a well-defined limit at infinity (ensuring that the problem of this paper is well-posed) and the components of the monopole and their derivatives satisfy asymptotic decay conditions near infinity, [14. In particular, $\left.\Phi\right|_{S_{\infty}^{2}}=\xi \in \mathrm{g}$ is the mass of the monopole.

More precisely, there exists a gauge in which

$$
\Phi=\xi+O\left(e^{-c r}\right)
$$

and $A=A_{w} d w+A_{\bar{w}} d \bar{w}+A_{r}$ such that

$$
A_{w} d w+A_{\bar{w}} d \bar{w}=A_{\infty}+O\left(e^{-c r}\right)
$$

for a $K_{\xi}$ connection $A_{\infty}$ on $S^{2}$, and

$$
A_{r}=O\left(e^{-c r}\right)
$$

as $r \rightarrow \infty$ and $c>0$ is a constant. There are similar estimates on the derivatives.

The asymptotic conditions on the monopole ensure along each radial geodesic the existence of a frame of fundamental solutions $g: \mathbb{R}^{+} \rightarrow G^{c}$ of the scattering equation

$$
\left(\partial_{r}^{A}-i \Phi\right) g=0
$$

with the property that $g(0) \in G$ and $\lim _{r \rightarrow \infty} g \exp (-i \xi r)$ is bounded. The solution is unique up to $g \mapsto g k$ for $k \in K_{\xi}$.

We can choose a family of solutions $g(w, r)$ to (6) that depend smoothly on $w$. It follows from the Bogomolny equation $F_{A}=* d_{A} \Phi$ that

$$
\left(\partial_{r}^{A}-i \Phi\right) \partial_{\bar{w}}^{A} g=0
$$

and $\partial_{\bar{w}}^{A} g \exp (-i \xi r)$ is bounded as $r \rightarrow \infty$, so

$$
\partial_{\bar{w}}^{A} g=g \eta(w)
$$

for some $\eta(w) \in \mathbf{p}_{\xi}$. In particular, $\partial_{\bar{w}} g(w, 0)=g(w, 0) \eta(w)$ since by the choice of coordinate system $\partial_{\bar{w}}^{A}=\partial_{\bar{w}}$ at $r=0$. Thus $g(w, 0)^{-1} \partial_{\bar{w}} g(w, 0) \subset \mathbf{p}_{\xi}$ and by (㺼) this means $g(w, 0): S^{2} \rightarrow G / K_{\xi}$ is a holomorphic map. In order to make sense of the value of $g$ at $r=0$ we have chosen a frame of the bundle there. This construction gives part of the following theorem.

Theorem 3.1. 12 The space of hyperbolic monopoles framed at $0 \in \mathbb{H}^{3}$ with gauge group $G$ and mass $\xi$ is diffeomorphic to the space of holomorphic maps $\mathrm{Hol}\left(S^{2}, G / H\right)$ where $H$ is the isotropy subgroup of $\xi$.

We can interpret the solution $g$ of (5) as a choice of gauge and then (6) and (7) give $(A, \Phi)$ with respect to this gauge, respectively showing that $A_{r}-i \Phi=0$ and $A_{\bar{w}}=\eta(w)$. We can choose another solution $g(w, r) p(w)$ of (6) for $p: \mathbb{C} \rightarrow P_{\xi}$ that has the same asymptotic properties as $g$ but no longer satisfies $g p(w, 0) \in G$ and with the property that with respect to this gauge

$$
A_{r}-i \Phi=0, A_{\bar{w}}=0 .
$$


This simply uses the fact that any holomorphic map $f: S^{2} \rightarrow G / K_{\xi}$ (locally) lifts to a map $u: \mathbb{C} \rightarrow G$, and there is a map $p: \mathbb{C} \rightarrow P_{\xi}$ such that up: $\mathbb{C} \rightarrow G^{c}$ is a lift of $f$ to an algebraic map. The maps $u$ and $p$ are unique up to $(u, p) \mapsto\left(u k, k^{-1} p\right)$ for $k: \mathbb{C} \rightarrow K_{\xi}$. The evaluation $g(w, 0)$ is of course a lift of a holomorphic map to $G / K_{x i}$.

Since $(A, \Phi)$ is Hermitian, with respect to the frame $g p$ satisfying $(\overline{6})$, the Hermitian metric $H=(g p)^{*} g p: \mathbb{H}^{3} \rightarrow G^{c} / G$ together with (8) gives the remainder of the monopole

$$
A_{r}+i \Phi=H^{-1} \partial_{r} H, A_{w}=H^{-1} \partial_{w} H
$$

The Bogomolny equations become $B(H)=0$ where

$$
B(H)=\sinh ^{2}(r) \partial_{r}\left(H^{-1} \partial_{r} H\right)+\left(1+|w|^{2}\right)^{2} \partial_{\bar{w}}\left(H^{-1} \partial_{w} H\right) .
$$

See [10] for further details.

Notice that $H(0)=p(w)^{*} p(w)$ is not well-defined (it depends on $w$ ) and $\left.H\right|_{S_{\infty}^{2}}$ is reduced i.e. $\left.H\right|_{S_{\infty}^{2}} \in K_{\xi}^{c} / K_{\xi}$ (for generic mass $K_{\xi}$ is a torus and $\left.H\right|_{S_{\infty}^{2}}$ is a potential.)

Now we represent a monopole as a Hermitian metric $H$ that satisfies (10). Given $H_{1}$ and $H_{2}$, define the endomorphism $h=H_{1}^{-1} H_{2}$.

Lemma 3.2. If $\Phi_{1}=\Phi_{2}$ on $S_{\infty}^{2}$, so the two monopoles have the same mass and we choose gauges in which the Higgs fields look the same, then the endomorphism $h$ is conjugate to a bounded endomorphism.

Proof. The Hermitian metric $H_{j}$ arises from $\left(A_{j}, \Phi_{j}\right)$ as $H_{j}=g_{j}^{*} g_{j}$. Put $\left.\Phi_{j}\right|_{S_{\infty}^{2}}=\xi$, then $g_{j}=G_{j}(w, r) \exp (i \xi r)$ for $G_{j}(w, r)$ bounded so $g_{2} g_{1}^{-1}$ is bounded. Now, $h=g^{-1}\left(\left(\left(g_{2} g_{1}^{-1}\right)^{*} g_{2} g_{1}^{-1}\right) g_{1}\right.$.

The two monopoles have the same asymptotic value precisely when $\left.h\right|_{S_{\infty}^{2}}=I$, the identity endomorphism. The complete metric on the space of Hermitian metrics given by taking the supremum over hyperbolic space of $\left\langle H^{-1} \delta H, H^{-1} \delta H\right\rangle$ uses the Killing form so the previous lemma implies that for two monopoles with the same mass, the distance between $H_{1}$ and $H_{2}$ is finite. Two monopoles are the same when the distance between their Hermitian metrics is zero.

\section{Approximate monopoles.}

In this section we will prove Theorem 11. Our strategy is as follows. For each holomorphic map and mass we can find an approximate monopole and a unique exact monopole nearby. The smaller the mass, the better the approximation. For any two holomorphic maps, the distance between the asymptotic values of the corresponding approximate monopoles is independent of the mass and positive. Thus, for small enough mass, when the two approximations are quite good, there must also be a positive distance between the asymptotic values of the two exact monopoles and the theorem is proven. We will actually use Hermitian metrics in place of monopoles since there is a good notion of distance between Hermitian metrics and there are techniques to estimate this distance.

A Hermitian metric $H: \mathbb{H}^{3} \rightarrow G^{c} / G$ can be associated to a more general set of pairs $(A, \Phi)$ than monopoles over $\mathbb{H}^{3}$. In fact, to any pair $(A, \Phi)$ that satisfies

$$
\left[\partial_{\bar{w}}^{A}, \partial_{r}^{A}-i \Phi\right]=0
$$


we can associate a Hermitian metric $H$ and $(A, \Phi)$ is retrieved from $H$ by (\$) and (9). This is the class of pairs we will consider.

Given $\xi \in \mathbf{g}$ and a holomorphic map $f: S^{2} \rightarrow G / K_{\xi}$ define

$$
\mathcal{H}_{f}=p^{*} \exp (2 i \xi r) p
$$

where $p: \mathbb{C} \rightarrow P_{\xi}$ is a map into the parabolic subgroup with the property that up is a lift of $f$ to an algebraic map from $\mathbb{C}$ to $G^{c}$ and $u$ is a lift of $f$ to a map from $\mathbb{C}$ to $G$. The map $u$ is ambiguous up to an action of $K_{\xi}$ on the right and hence $p$ inherits this ambiguity on the left. The expression for $\mathcal{H}_{f}$ is independent of this ambiguity.

In order to show the existence of a monopole for any given mass and holomorphic map we use the non-linear heat flow for Hermitian metrics with initial value given by $\mathcal{H}_{f}$.

$$
H^{-1}(w, r, t) \partial H(w, r, t) / \partial t=B(H(w, r, t)), H(w, r, 0)=\mathcal{H}_{f}(w, r)
$$

Theorem 4.1. 110, 12] There is a unique solution $H(w, r, t)$ of (19).

The solution $H(w, r, t)$ of the heat flow converges to a Hermitian metric that satisfies $B(H(w, r, \infty))=0$ and gives rise to a monopole with holomorphic map $f$. Together with the scattering construction described in Section 3 this gives the proof of Theorem 3.1.

It is worth pointing out that the construction of monopoles from holomorphic maps is treated differently in $[10]$ and $\sqrt{12}$ and here it is treated slightly differently again. In 10] the initial choice of Hermitian metric used explicitly known symmetric hyperbolic monopoles. In [12], since both instantons and hyperbolic monopoles were treated together it was more convenient to choose an initial Hermitian metric that was independent of such information (and also to use something more general than a Hermitian metric.) Neither of these suffice for our purposes here. In order that the limiting connections at infinity of different monopoles can be compared we need to ensure that a common reduction of the monopoles to a subgroup (usually a maximal torus) is used. This is why the parabolic subgroup is specified and features in the Hermitian metric above. In particular, the approximate monopole defined by $\mathcal{H}_{f}$ has the same asymptotic mass as the monopole associated to $H(w, r, \infty)$, so $d\left(H(w, r, \infty), \mathcal{H}_{f}\right)$ is finite.

The metric on the space of Hermitian metrics is given by $\operatorname{tr}\left(H^{-1} d H\right)$ so the heat flow gives an estimate of the distance from the initial $\mathcal{H}_{f}$ and the final $H(w, r, \infty)$ :

$$
\begin{aligned}
d\left(H(w, r, \infty), \mathcal{H}_{f}\right) & \leq \int_{0}^{\infty}|B(H(w, r, t))| d t \\
& \leq \int_{0}^{\infty} \max _{|\rho|=s}\left|B\left(\mathcal{H}_{f}(w, \rho)\right)\right|^{2} G(s, r) d s
\end{aligned}
$$

where the second inequality comes from the fact that $|B(H(w, r, t))|$ is dominated by a solution of the linear heat flow for a Laplacian like operator that reduces to the usual Laplacian on radially symmetric functions. When we maximise $\left|B\left(\mathcal{H}_{f}\right)\right|$ over spheres of constant radius we get a function of the radius so we can use a one-dimensional Green's function $G(s, r)$. See [10] 12] for the proof of this and also 3. 22 where this technique is introduced.

The following two propositions estimate how well (11) approximates a monopole by using (14). The first proposition is enough to prove Theorem 1. We go on to 
prove more in the second proposition. It relates the estimate in the first proposition with $\left|f^{*} \omega\right|$, the two-form on $S^{2}$ pulled back by the holomorphic map, where $f^{*} \omega$ is compared to the standard two-form on $S^{2}$ to get its magnitude. This precise information is included particularly to show how good the approximation is as the holomorphic maps bubble.

Proposition 4.2. $d\left(H(w, r, \infty), \mathcal{H}_{f}\right) \leq C|\xi| \max _{S_{\infty}^{2}}\left|F_{A}\right|$.

Proof. By (14) we have to get an upper bound for $|B(\mathcal{H}(w, r))|$ on each sphere $r=$ constant. Using (8) and (9) with $H=\mathcal{H}_{f}$, and going to a unitary gauge we get

$$
\Phi=\xi, A_{r}=0, A_{\bar{w}}=\exp (i \xi r) u^{-1} \partial_{\bar{w}} u \exp (-i \xi r), A_{w}=\exp (-i \xi r) u^{-1} \partial_{w} u \exp (i \xi r)
$$
and $B(\mathcal{H}(w, r))=-i\left(1+|w|^{2}\right)^{2} F_{\bar{w} w}$.

The connection $A$ splits into a radially independent $K_{\xi}$ connection and an exponentially decaying connection. More precisely, put

$$
\mathbf{g}^{c}=\mathbf{k}_{\xi} \oplus \mathbf{n}_{\xi}^{+} \oplus \mathbf{n}_{\xi}^{-}
$$

where $\mathbf{k}_{\xi}=\operatorname{ker}[\xi, \cdot]$ is the Lie algebra of $K_{\xi}$ and $\mathbf{k}_{\xi} \oplus \mathbf{n}_{\xi}^{+}=\mathbf{p}_{\xi}$, the Lie algebra of $P_{\xi}$. Alternatively, we can characterise the sub-spaces by

$$
(\exp (i \xi r)-I) \cdot \mathbf{k}_{\xi}=0=\lim _{r \rightarrow+\infty} \exp ( \pm i \xi r) \cdot \mathbf{n}^{ \pm}
$$

Decompose $v \in \mathbf{g}^{c}$ accordingly as $v=v^{0}+v^{+}+v^{-}$.

The connection $A$ decomposes as $A=A^{0}+a$ for $a=A_{\bar{w}}^{+} d \bar{w}+A_{w}^{-} d w$ with the property that $A^{0}$ is a $K_{\xi}$ connection independent of $r$, and $a$ is a 1-form that decays exponentially as $r \rightarrow \infty$. Then $F_{\bar{w} w}=F_{A^{0}}+d_{A^{0}} a+a \wedge a$ and $F_{A^{0}}$ is independent of $r$ whilst the rest decays exponentially, so $F_{A^{0}}=\left.F_{A}\right|_{S_{\infty}^{2}}$.

Define $c_{\xi}>0$ to be the smallest eigenvalue of the action of $\exp (i \xi r)$ on $\mathbf{g}^{c}$. Then each time we say that a term decays exponentially, it decays at least as fast as $e^{-c_{\xi} r}$. Notice that $c_{\xi} \leq|\xi|$. We have $\left|F_{A}\right| \leq M_{1}\left|F_{A^{0}}\right|\left(1-e^{-c_{\xi} r}\right)$ for some constant $M_{1} \geq 1$ and thus

$$
\begin{aligned}
d\left(H(w, r, \infty), \mathcal{H}_{f}\right) & \leq \int \max _{|\rho|=s}|B(\mathcal{H})(w, \rho)|^{2} G(s, r) d s \\
& \leq M_{1} \max _{S_{\infty}^{2}}\left|F_{A}\right| \int_{0}^{\infty} \frac{\left(1-e^{-c_{\xi} s}\right) \min \{r, s\}}{\sinh ^{2} s} d s
\end{aligned}
$$

where $\min \{r, s\} / \sinh ^{2} s$ is the one-dimensional Green's function.

Now

$$
\int_{0}^{\infty} \frac{\left(1-e^{-c_{\xi} s}\right) \min \{r, s\}}{\sinh ^{2} s} d s=\int_{0}^{r} \frac{\left(1-e^{-c_{\xi} s}\right) s}{\sinh ^{2} s} d s+r \int_{r}^{\infty} \frac{\left(1-e^{-c_{\xi} s}\right)}{\sinh ^{2} s} d s
$$

and the second term of the right hand side converges to 0 as $r \rightarrow \infty$. Since $1-e^{-c_{\xi} s} \leq c_{\xi} s$, the first term is dominated by $c_{\xi} M_{2}$ for a constant $M_{2}$. Since $c_{\xi} \leq$ $|\xi|$ the proposition follows. The constant $C$ in the statement of the proposition does depend on the holomorphic map $f$, and is bounded below by a constant independent of $f$.

In particular, the estimate depends only on the holomorphic map and the mass. For small mass, the distance is small. For any two holomorphic maps $f$ and $g$, notice that restricted to $S_{\infty}^{2}$ the distance $d\left(\mathcal{H}_{f}, \mathcal{H}_{g}\right)$ is independent of the mass since it depends on

$$
\mathcal{H}_{f}^{-1} \mathcal{H}_{g}=p_{f}^{-1} \exp (-2 i \xi r)\left(p_{f}^{*}\right)^{-1} p_{g}^{*} \exp (2 i \xi r) p_{g}
$$


which is independent of $\xi$ in the limit $r \rightarrow \infty$. Of course the holomorphic maps $f$ and $g$ use $\xi$ to be defined, but they could equally well use a mass that gives the same homogeneous manifold, like $\lambda \xi$ for $\lambda \in \mathbb{R}^{*}$.

Thus, if we take any two holomorphic maps and choose the mass small enough, then the nearby monopoles must have different asymptotic values. This argument extends to two disjoint compact subsets of the space of holomorphic maps and Theorem 1 is proven.

Proposition 4.3. $\left.\left\langle F_{A}, \Phi\right\rangle\right|_{S_{\infty}^{2}}=f^{*} \omega$.

Proof. In the proof of the previous proposition we saw that $F_{A}$ has a radially independent part $\left.F_{A}\right|_{S_{\infty}^{2}}$ and an exponentially decaying part. Since $F_{\bar{w} w}$ vanishes at $r=0$ we get an identity relating $\left.F_{A}\right|_{S_{\infty}^{2}}$ with the term that cancels it.

In the notation of the previous proof, $F_{\bar{w} w}^{0} d \bar{w} d w=\left.F_{A}\right|_{S_{\infty}^{2}}+\left[A_{\bar{w}}^{+}, A_{w}^{-}\right]^{0} d \bar{w} d w$ so

$$
\left.\left\langle F_{A}, \Phi\right\rangle\right|_{S_{\infty}^{2}}=\left.\left\langle-\left[A_{\bar{w}}^{+}, A_{w}^{-}\right]^{0} d \bar{w} d w, \Phi\right\rangle\right|_{r=0}=\left.\left\langle-\left[A_{\bar{w}}^{+}, A_{w}^{-}\right] d \bar{w} d w, \Phi\right\rangle\right|_{r=0}
$$

where the last equality follows from the fact that $\Phi$ is orthogonal to $\mathbf{n}^{ \pm}$. But at $r=0, A_{\bar{w}}=u^{-1} \partial_{\bar{w}} u, A_{w}=u^{-1} \partial_{w} u$ so

$$
\left.\left\langle F_{A}, \Phi\right\rangle\right|_{S_{\infty}^{2}}=-\left\langle\left[u^{-1} \partial_{\bar{w}} u, u^{-1} \partial_{w} u\right], \xi\right\rangle d \bar{w} d w=f^{*} \omega .
$$

The previous proposition shows that the heat flow gives bad estimates for wellseparated monopoles. That is, if a sequence of holomorphic maps bubble then the pull-back of the Kahler form will bubble and $\left\langle F_{A}, F_{A}\right\rangle$ which determines the accuracy of the approximate monopole, gets a contribution from $\left\langle F_{A}, \Phi\right\rangle$, and hence gets large. (It may even be true that $\left\langle F_{A}, \Phi\right\rangle$ controls $\left\langle F_{A}, F_{A}\right\rangle$.)

Well-separated monopoles are Dirac-like and are the source of our intuition that hyperbolic monopoles have interesting asymptotic limits. So far we have not been able to produce good approximate well-separated monopoles. It would be very interesting to see such approximations since they would combine the linear nature of Dirac monopoles with the soliton nature of gluing together holomorphic maps.

Given the intuition that asymptotic values of well-separated monopoles look like asymptotic values of Dirac hyperbolic monopoles, we would be able to relax the condition in Theorem 1 allowing one set to be non-compact and in particular apply the theorem to a point and a deleted neighbourhood of the point.

Conjecture 2. Given a compact subset and a disjoint subset in the parameter space of monopoles, if the mass is small enough then the asymptotic values of the corresponding hyperbolic monopoles respectively give two distinct subsets.

A related and interesting issue is to know if the set of monopoles with bounded curvature on $S_{\infty}^{2}$ and bounded mass, gives rise to a compact set in the space of holomorphic maps. Such a result would also prove the conjecture. In special cases it can be shown that for a fixed holomorphic map the maximum value of the curvature at infinity is a monotone decreasing function of the mass so the conjecture follows.

\section{Comparison with Euclidean monopoles.}

The asymptotic value of a Euclidean monopole gives a symmetric connection on the sphere at infinity, and in particular, all monopoles (with the same mass and charge) give rise to the same connection at infinity. This contrasts with the 
hyperbolic case and it is interesting to see where the point of departure from the behaviour of hyperbolic monopoles occurs.

The proof of Proposition 4.2 goes through for Euclidean monopoles with the only change being in the one-dimensional Green's function. We use $\min \{r, s\} / s^{2}$ instead of $\min \{r, s\} / \sinh ^{2} s$. Now

$$
d\left(H(w, r, \infty), \mathcal{H}_{f}\right) \leq C \max _{S_{\infty}^{2}}\left|F_{A}\right|\left(\int_{0}^{r} \frac{\left(1-e^{-c_{\xi} s}\right)}{s} d s+r \int_{r}^{\infty} \frac{\left(1-e^{-c_{\xi} s}\right)}{s^{2}} d s\right)
$$

and the second term is bounded whilst the first term is $O(\ln r)$. This is enough to show that the heat flow converges and thus Theorem 3.1 is true for Euclidean monopoles, 10, 19. But we see that the asymptotic value of the monopole can move arbitrarily far and a posteriori we know that all of the asymptotic values converge to the same connection.

This is an appropriate place to mention the result of Murray and Singer [16] regarding asymptotic values of hyperbolic monopoles. They show that an $S U(n)$ hyperbolic monopole is determined by $\left(\left.\nabla^{0}\right|_{S_{\infty}^{2}}\right)^{0,1}$ and $\left(\left.b_{+}\right|_{S_{\infty}^{2}}\right)^{0,1}$, using their notation. The first term is the asymptotic value of the monopole and the second term is an artifact of the holomorphic gauge they use giving off-diagonal terms. In the good unitary gauge defined by Rade [20] the asymptotic value is a $U(1)^{n}$ connection. The term $\left(\left.b_{+}\right|_{S_{\infty}^{2}}\right)^{0,1}$ essentially encodes the holomorphic map which is also enough to give a Euclidean monopole so no new behaviour is seen there. It is not so surprising since their methods are similar to those applied to Euclidean monopoles.

Finally, we mention a maximum principle which a priori may have led to a proof that hyperbolic monopoles are determined by their asymptotic values. It ends up that the maximum principle also applies to Euclidean monopoles so it proves a result that is true for both cases.

As in Section 3 define $h=H_{1}^{-1} H_{2}$ for two Hermitian metrics satisfying $B\left(H_{i}\right)=$ 0 . Consider $\sigma(h)=\operatorname{tr}(h)+\operatorname{tr}\left(h^{-1}\right)-2 n,(n=\operatorname{tr} I)$. This is a non-negative quantity that vanishes precisely when $H_{1}=H_{2}$. The problem of showing that a monopole is determined by its asymptotic values then becomes the problem of showing that if the asymptotic value of $\sigma(h)$ vanishes then $\sigma(h)$ vanishes identically. The following inequality leads to a maximum principle.

$$
\sinh ^{2}(r) \partial_{r}^{2} \sigma+\left(1+|w|^{2}\right)^{2} \partial_{\bar{w}} \partial_{w} \sigma \geq 0 .
$$

It applies to Euclidean monopoles also

$$
r^{2} \partial_{r}^{2} \sigma+\left(1+|w|^{2}\right)^{2} \partial_{\bar{w}} \partial_{w} \sigma \geq 0 .
$$

The maximum principle states that $\sigma(h)$ is dominated by any function that dominates $\sigma(h)$ on the boundary and lies in the kernel of the second order partial differential operator above. The important point here is that there are two boundary components, $r=0$ and $r=\infty$, since $\sigma(h)$ depends on $w$ at $r=0$. The function $a+b r$ is a good comparison function for constants $a$ and $b$ chosen so that $a \geq \max _{r=0} \sigma(h)$ and $b>0$. As $b \rightarrow 0$ we see that

$$
\max _{r=0} \sigma(h) \geq \max _{r=\infty} \sigma(h) .
$$

This is true of both hyperbolic monopoles and Euclidean monopoles. In the latter case, $\max _{r=\infty} \sigma(h)=0$ so the inequality is trivial. 


\section{REFERENCES}

[1] M.F. Atiyah. Instantons in two and four dimensions. Comm. Math. Phys., 93, 437-451 (1984).

[2] D.M. Austin and P.J. Braam. Boundary values of hyperbolic monopoles. Nonlinearity, 3, 809-823 (1990).

[3] S.K. Donaldson. Anti-self-dual Yang-Mills connections over complex algebraic surfaces and stable vector bundles. Proc. LMS, 30, 1-26 (1985).

[4] S.K. Donaldson. Nahm's equations and the classification of monopoles. Comm. Math. Phys., 96, 387-407 (1984).

[5] H. Garland and M.K. Murray. Kac-Moody monopoles and periodic instantons. Comm. Math. Phys., 120, 335-351 (1988).

[6] Richard S. Hamilton. Harmonic maps of manifolds with boundary. Lecture Notes in Math. 471, Springer, New York, 1975.

[7] N.J. Hitchin. On the construction of monopoles. Comm. Math. Phys., 89, 145-190 (1983).

[8] N.J. Hitchin. A new family of Einstein metrics. Manifolds and geometry (Pisa, 1993), 190-222, Sympos. Math., XXXVI, Cambridge Univ. Press, Cambridge, 1996.

[9] A. Jaffe and C.H. Taubes. Vortices and monopoles. Birkhäuser, Boston, 1980.

[10] Stuart Jarvis. Monopoles to rational maps via radial scattering. Preprint, (1996).

[11] Stuart Jarvis and Paul Norbury. Compactification of hyperbolic monopoles. Nonlinearity, 10, 1073-1092 (1997).

[12] Stuart Jarvis and Paul Norbury. Degenerating metrics and instantons on the four-sphere. J. Geom. Phys., 27, 79-98 (1998).

[13] Stuart Jarvis and Paul Norbury. Zero and infinite curvature limits of hyperbolic monopoles. Bull. LMS, 29, 737-744 (1997).

[14] Rafe Mazzeo and Johan Rade. Private communication.

[15] Michael Murray and Michael Singer. On the complete integrability of the discrete Nahm equations. To appear in Comm. Math. Phys. (1999)

[16] Michael Murray and Michael Singer. Spectral curves of hyperbolic monopoles. Nonlinearity, 9, 973-997 (1996).

[17] Werner Nahm. Self-dual monopoles and calorons. Lecture Notes in Phys. 201, Springer, Berlin, 189-200 (1983).

[18] Werner Nahm. The construction of all self-dual multimonopoles by the ADHM method. In Monopoles in quantum field theory (Trieste), World Sci. Pub., pages 87-94, 1981.

[19] Paul Norbury. Periodic instantons and the loop group. http://xxx.adelaide.edu.au/abs/math$\mathrm{ph} / 9902007$

[20] Johan Rade. Singular Yang-Mills fields. Local theory. I. J. Reine Angew. Math. 452 (1994), $111-151$

[21] L.M. Sibner and R.J. Sibner. Classification of singular Sobolev connections by their holonomy. Commun. Math. Phys., 144, 337-350 (1992).

[22] Carlos T. Simpson. Constructing variations of Hodge structure using Yang-Mills theory and applications to uniformization. Jour. Amer. Math. Soc., 1, 867-918 (1988).

Department of Mathematics and Statistics, The University of Melbourne, Victoria, Australia 3010.

E-mail address: norbs@ms.unimelb.edu.au 